\title{
Defects in Aortic Fusion and Craniofacial Vasculature in the Holoprosencephalic Mouse Embryo under Inhibition of Sonic Hedgehog Signaling
}

\author{
Takashi Nagase, MD, PhD, ${ }^{*}$ Miki Nagase, MD, PhD, ${ }^{\dagger}$ Kotaro Yoshimura, MD, PhD, ${ }^{\ddagger}$ \\ Masafumi Machida, MD, PhD, ${ }^{*}$ Masaaki Yamagishi, MD, PhD*
}

Tokyo, Japan

Sonic hedgehog (Shh) is a well-known morphogen indispensable in facial and nervous development, and recently it has also garnered much attention as a potent angiogenic factor. We previously created an animal model of holoprosencephaly by administration of cyclopamine, a specific inhibitor of hedgehog signaling, to the mouse embryos cultured in vitro, and found several types of angiogenic defects. In this study, we focused on other angiogenic phenotypes in the same model. When cyclopamine was added for embryonic day (E) 8.0-9.5, a pair of immature dorsal aortae, which normally fuse to form the single aorta by E9.5, remained to be separated. Expressions of vascular endothelial growth factor and bone morphogenetic protein 4 , putative mediators of aortic fusion, were also reduced around the aorta by blockade of Shh signaling. When cyclopamine was added for E8.5-10.5, vessels on the surface of craniofacial region (possibly external cardinal veins) were extended and malformed. These results suggest that Shh signaling is essential for some aspects of embryonic angiogenesis, and that pathophysiology of

From the *Clinical Research Center, National Hospital Organization Murayama Medical Center, 2-37-1 Gakuen, MusashimurayamaCity, Tokyo 208-0011, Japan; the ${ }^{\dagger}$ Department of Nephrology and Endocrinology, and 'Department of Plastic and Reconstructive Surgery, University of Tokyo Graduate School of Medicine, 7-3-1 Hongo, Bunkyo-ku, Tokyo 113-8655, Japan.

Address correspondence and reprint requests to Takashi Nagase, MD, PhD, Clinical Research Center, National Hospital Organization Murayama Medical Center, 2-37-1 Gakuen, Musashimurayama-City, Tokyo 208-0011, Japan; E-mail: nagase-t@ murayama-crc.jp

We thank Prof. Isao Koshima for his support for our experiments. This work was supported by a Grant-In Aid for Scientific Research from the Japanese Ministry of Education, Science and Culture given to T. N. (\#15390537). holoprosencephaly may involve, at least in part, the Shh-dependent angiogenesis.

Key Words: Sonic hedgehog, aorta, angiogenesis, cyclopamine, whole embryo culture

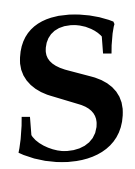

onic hedgehog (Shh) is a well-known morphogen in embryogenesis, mediating development of various tissues and organs such as the central nervous system, face, limb, vessels, gastrointestinal tract and skin appendages. ${ }^{1}$ As for craniofacial malformation, Shh gene mutation is known to be responsible for familial holoprosencephaly. ${ }^{2,3}$ Shh gene targeting in mouse embryos result in severe holoprosencephalic phenotypes such as cyclopia. ${ }^{4}$ In contrast, hypertelorism-like phenotype can be observed by overexpression of Shh in the cranial region of chicken embryos. ${ }^{5}$ Chick or mouse embryos exposed to excess ethanol (animal models of craniofacial anomalies due to fetal alcohol syndrome) exhibited expression changes of Shh-related genes. ${ }^{6,7}$ These lines of evidences indicate indispensable roles of Shh in craniofacial development and anomalies.

We previously reported a mouse embryonic model of holoprosencephaly by administration of cyclopamine, a specific inhibitor for hedgehog signaling, using whole embryo culture system. ${ }^{8}$ In our model, the embryos were treated with $20 \mu \mathrm{M}$ cyclopamine for embryonic day (E) 8.5-10.5. The angle and distance between the two nasal placodes were reduced in the cyclopamine-treated embryos, probably showing mild phenotype of holoprosencephaly. ${ }^{8}$ The reason why our phenotype was milder than the cyclopia in the Shh knockout mice may be that the time window of Shh signaling blockade was 
shorter and more specific to the stage of craniofacial development in our model. ${ }^{8}$ If this consideration is correct, the phenotype should be more general and severe when Shh signaling is blocked earlier, as Cordero et $\mathrm{al}^{9}$ suggested in chick embryos.

It should also be kept in mind that the period around E8 -10 is characterized by craniofacial as well as cardiovascular development. The heart primordium appears at around E8, and pulsation can be detected under microscope at around E9. Furthermore, morphogenesis of the aorta is accomplished through dynamic remodeling process of fusion of the two separate dorsal aortae into one. ${ }^{10,11}$ In the mouse embryos, a pair of dorsal aortae begin to fuse later than $\mathrm{E} 8.5,{ }^{12}$ and the aorta is fused at the limb bud level by E9.5, whereas the rostral aortic arches are still separated forming a figure Y-shape. ${ }^{13,14}$ These facts suggest that the craniofacial malformations originated in this embryonic stage may involve defects of cardiovascular systems.

Indeed, Shh induced vasculo-angiogenesis has become a recent hot topic among vascular biologists, suggesting possible involvement of abnormal angiogenesis in the pathogenesis of holoprosencephaly. For example, Shh mediates early-stage vasculogenesis in the chicken and mouse embryos. ${ }^{15}$ Shh promotes angiogenesis of ischemic limbs or muscles in the adult mice, ${ }^{16,17}$ and rescues peripheral neuropathy of diabetic mice via augmenting vascularization of the nerve. ${ }^{18}$ In zebrafish embryos, Shh mediates morphogenesis of the aorta by inducing vascular endothelial growth factor (VEGF) in the mesoderm. ${ }^{19}$ We also reported previously defects of angiogenesis in the neural tube ${ }^{20}$ and the yolk $\operatorname{sac}^{21}$ of the cultured embryos under blockade of hedgehog signaling.

In this study, we focused on other vascular phenotypes caused by inhibition of Shh signaling: aortic fusion defects and abnormal craniofacial vessels. These vascular phenotypes, as well as severity of craniofacial morphology, were dependent on the timing and duration of cyclopamine treatment. Possible roles of Shh-dependent angiogenesis in the pathogenesis of holoprosencephaly are also discussed.

\section{MATERIAls AND Methods}

M ouse whole embryo culture: Mouse whole embryo culture was performed as described previously. ${ }^{8,20-22}$ Cyclopamine (TRC, Toronto, Canada) stock (20 $\mathrm{mM}$ dissolved in ethanol) or jervine (TRC) stock (10 $\mathrm{mM}$ in ethanol) was added to the rat serum (the medium, specially purchased from Charles River Japan, Kanagawa, Japan), yielding $20 \mu \mathrm{m}$ of cyclopamine or jervine. Ethanol alone was added in the control group. Mouse embryos of E7.8 - 8.5 were dissected out from pregnant ICR mice (Clea, Tokyo, Japan) under anesthesia with ether and cultured using the whole embryo incubator (Ikemoto Rika, Tokyo, Japan) until the stage of E8.5-10.5, according to a standard protocol of oxygenation. ${ }^{23}$ The collected embryos were fixed overnight in $4 \%$ paraformaldehyde and cryosectioned in $14 \mu \mathrm{m}$ thickness.

Immunohistochemistry: Whole-mount immunohistochemistry was carried out as described previously. ${ }^{21}$ Briefly, the fixed whole embryos were incubated with 1:100 solution of biotinylated antiPECAM-1 antibody (MEC13.3; BD Pharmingen, San Diego, CA). Subsequently, samples for PECAM-1 staining were reacted using a biotinyl tyramide amplification kit (PerkinElmer Life Sciences, Boston, MA). Immunoreactivity was visualized with diaminobenzidine.

Cryosections for PECAM-1 staining were incubated with biotinylated anti-PECAM-1 antibody (1:100) and subjected to tyramide amplification system. ${ }^{20,21}$ Signals were detected using a metal enhanced diaminobenzidine kit (Pierce, Rockford, IL).

In situ hybridization: cDNA probes for mouse Shh, Ptc-1, Gli-1 and VEGF were described previously. ${ }^{8,20} \mathrm{~A}$ cDNA probe for mouse bone morphogenetic protein 4 (BMP-4) (X56848; bases 362 to 1011) was cloned by RTPCR. The obtained cDNA fragment was subcloned into pBluescript II SK${ }^{-}$and sequenced using an $\mathrm{ABI}$ PRISM 310 Genetic Analyzer (Applied Biosystems, Foster City, CA) to confirm their authenticity.

In situ hybridization on frozen sections was performed as previously described. ${ }^{8,20,22}$ Briefly, cryosections were treated with proteinase $\mathrm{K}(0.3 \mu \mathrm{g} / \mathrm{mL})$ at $37^{\circ} \mathrm{C}$ for $2 \mathrm{~min}$, and hybridized with digoxigeninlabeled antisense cRNA probes at $60^{\circ} \mathrm{C}$. After washing, the slides were incubated with anti-digoxigenin Fabalkaline phosphatase conjugate. The hybrids were detected with nitro blue tetrazolium and 5-bromo-4chloro-3-indolyl phosphate.

\section{RESULTS}

\section{General and Craniofacial Morphology was More Severely Affected when Shh Signaling was Blocked Earlier}

$\mathbf{W}$ e previously reported that mild holoprosencephaly-like phenotype was obtained if cyclopamine was administered from E8.5 in the whole embryo culture system. ${ }^{8}$ We first examined the spectrum of the morphological phenotype caused by cyclopamine administered at various timing, considering that the phenotype may be more severe if cyclopamine was added earlier as reported in chick embryos by Cordero et al. ${ }^{9}$ 
If cyclopamine was administered to the embryos from somite stages $0-1$ (about E7.8) and the embryos were cultured till E9.5, the whole development was drastically impaired (Fig 1A). Morphogenesis of the head was so inhibited that it could hardly be recognized as a head (Fig 1E). The malformation was slightly milder if cyclopamine was added from somite stages $4-5$ (about E8.0), showing defects in conformational rotation and underdevelopment of the brain region (Figs $1 \mathrm{~B}$ and $\mathrm{F}$ ). The embryonic rotation was mostly normal under cyclopamine treatment from somite stages 8-9 (about E8.3-8.5, Fig 1C), and the milder holoprosencphaly-like anomaly was observed as seen in our previous study ${ }^{8}$ (Fig 1G).

These results indicated that a wide spectrum of severity in the craniofacial phenotype, as seen in clinical holoprosencephaly cases, can be caused in our model by only a half day of time difference in starting cyclopamine administration. In other words, it was suggested that the mode of morphogenic action of Shh signaling may be considerably different between the stages of E8.0 and E8.5. We shall describe the different vascular phenotypes between these two stages in the following sections.

\section{Aortic Fusion was Impaired by Blockade of Shh Signaling for E8.0-9.5}

We examined vascular phenotypes of the cultured mouse embryos in which hedgehog signaling was blocked from E8.0 (somite stages $4-5$ and thereafter). In this condition, we previously reported defective angiogenesis in the yolk sacs, which was turned out to be dependent on Indian hedgehog, not Sonic hedgehog. ${ }^{21}$ Thus, we focus in this paper on angiogenesis within the embryos themselves.

When the embryos were collected at E8.5, gross anatomy of the embryos could not be distinguished between the control and the cyclopamine-treated embryos (Figs 2A, C). In the whole mount immunostaining with anti-PECAM-1 antibody, a marker for vascular endothelium, a pair of dorsal aortae could be clearly recognized and their fusion had not started yet in the control and cyclopamine-treated embryos (Figs 2B, D), as reported in the normal E8.5 embryos. ${ }^{12}$

When the cultured embryos were collected at E9.5, the shape of the control embryos was similar to normal E9.5 embryos (Fig 2E), and the aorta was fused at the limb bud level (Fig 2F). In contrast, size of the embryo was remarkably reduced and turning was incomplete in the cyclopamine-treated groups (Fig 2G). PECAM-1 whole mount immunostaining revealed that the paired dorsal aortae remained unfused in this group (Fig $2 \mathrm{H}$ ). To confirm that this phenotype is caused by specific inhibition of hedgehog signaling, we also administered another specific inhibitor jervine. We found that there were no difference between cyclopamine and jervine treated embryos in both the E8.5 and E9.5 stages. We therefore
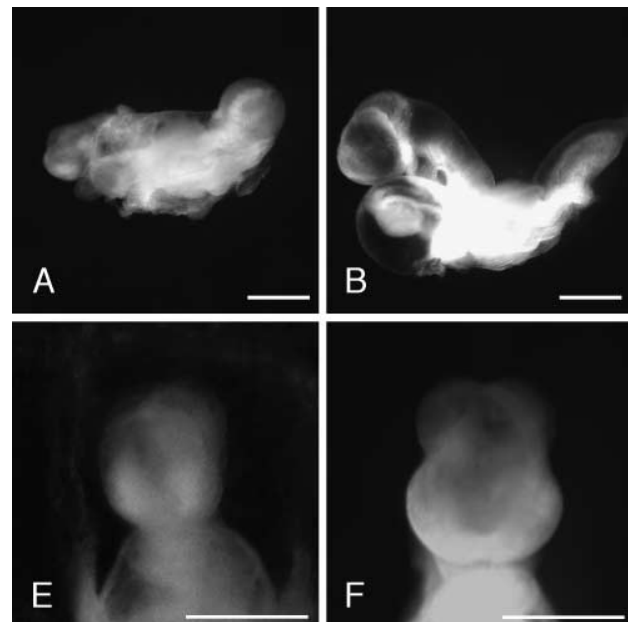

Somite 1/2-

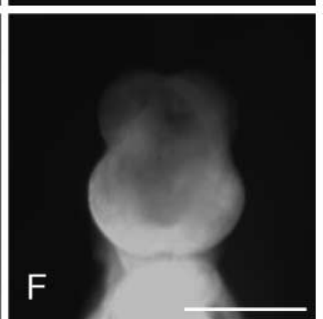

Somite 4/5-
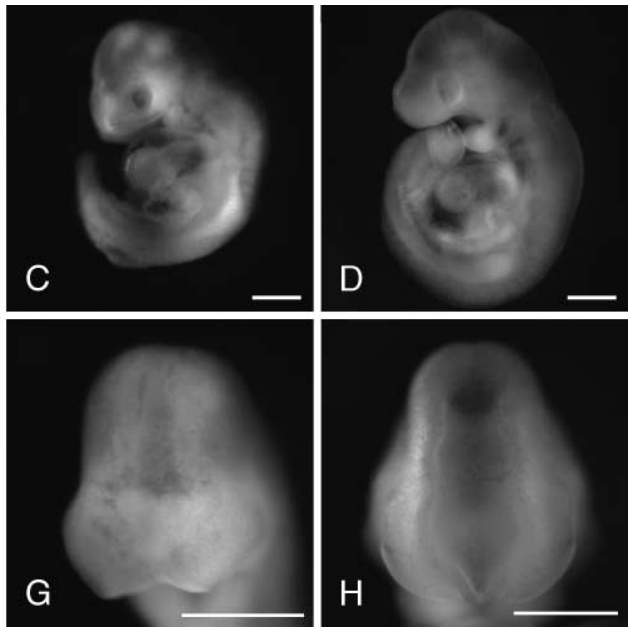

Somite 8/9-

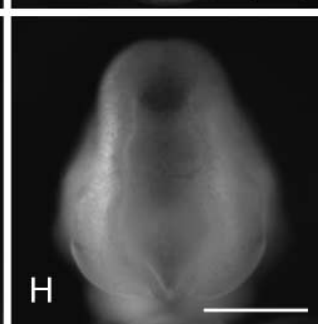

Control

Fig 1 Time dependent variation of general and craniofacial morphology in the mouse embryos cultured in vitro with or without hedgehog blocker cyclopamine. Whole embryo culture and $20 \mu \mathrm{M}$ cyclopamine administration are started at developmental stages of somite $0 / 1$ (A, E), somite $4 / 5$ (B, F) and somite $8 / 9(\mathrm{C}, \mathrm{G})$. The control embryos are cultured from somite $4 / 5$ stage without cyclopamine $(\mathrm{D}, \mathrm{H})$. All the embryos are collected at E9.5. Note that the malformation was more severe if cyclopamine treatment was started earlier. Bars, $500 \mu \mathrm{m}$. 


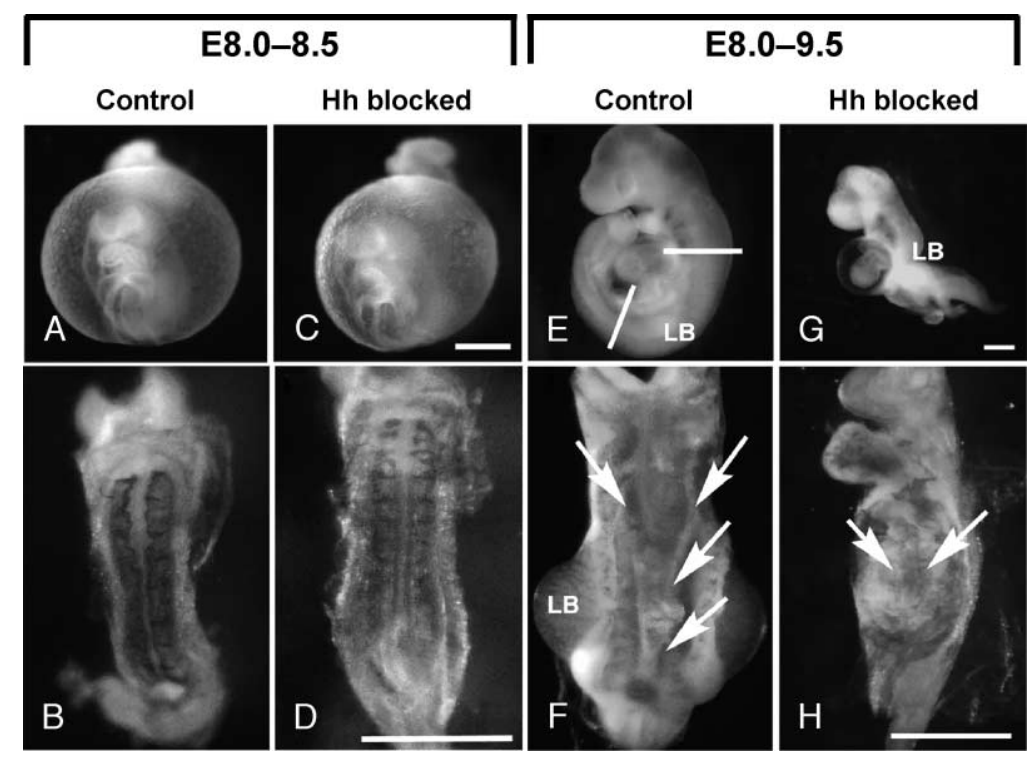

Fig 2 Administration of hedgehog blocker cyclopamine or jervine impairs the aortic fusion. The embryos are cultured from E8.0, collected at E8.5 (A-D) and E9.5 (E-H), and immunostained with anti-PECAM-1 antibody (B, D, F, H). (A) The E8.5 control embryos cultured with ethanol only exhibit normal morphology. The embryo is about somites stage 10 and observed within a yolk sac before turning. (B) Whole mount PECAM-1 immunostaining of the E8.5 control embryos shows the paired dorsal aortae, as seen in the normal embryo11. (C, D) The E8.5 embryos cultured with cyclopamine or jervine show the same gross morphology $(\mathrm{C})$ and the same structure of the PECAM-1 positive paired dorsal aortae (D) as the control group. (E) The E9.5 control embryo shows normal morphology. (F) The ventral view of the E9.5 control embryo immunostained with anti-PECAM-1 antibody. The head and tail are cut off at the white lines indicated in (E), and the ventral endodermal tissues are also removed. Note the PECAM-1 positive Y-shaped aorta (arrows), indicating that the aorta has been fused at the limb bud (LB) level, but still separated in the rostral aortic arches. (G) General growth is grossly retarded in the E9.5 embryos cultured with cyclopamine or jervine. Note that turning is incomplete in this group. (F) PECAM-1 immunostaining of the E9.5 cyclopamine/jervine treated embryos shows that the paired dorsal aortae are still separated (arrows) at the limb bud level, as seen in E8.5. Bars, $500 \mu \mathrm{m}$.

examined only the cyclopamine-treated embryos in the following analyses.

To observe the aortic fusion more clearly, we sectioned the E9.5-cultured embryos at the limb bud level and performed PECAM-1 immunohistochemistry. Structure of the fused aorta was normal in the control group (Fig 3A). On the other hand, two dorsal aortae could be detected and the fusion did not occur in the cyclopamine-treated embryos (Fig 3B). These results indicated that administration of Hedgehog signaling blockers at E8.0 resulted in impairment of the fusion process of the two dorsal aortae.

\section{Expressions Changes of Vegf and Bmp-4 Were Associated with the Aortic Fusion Defects by Cyclopamine}

We further investigated alterations of gene expressions associated with the morphological changes in the cultured embryos with cyclopamine during E8.0-9.5.
Initially, we planned to confirm the blocking effect of hedgehog signaling by cyclopamine in our experimental protocol. As the expressions of Ptc- 1 and Gli-1 are directly regulated by Hedgehog signaling itself, ${ }^{24}$ inhibition of Hedgehog signaling can be monitored by downregulation of these genes. ${ }^{8,20}$ Indeed, we observed remarkable downregulation of Ptc-1 and Gli-1 expressions, but not Shh expression, in the cyclopaminetreated embryos by in situ hybridization sectioned at the limb bud level (Figs 3C-H).

To obtain molecular insights into mechanisms of the aortic fusion, we further planned to investigate expression changes of putative downstream genes. Lawson et $\mathrm{al}^{19}$ reported that Shh-dependent morphogenesis of the aorta in zebrafish embryos is mediated by VEGF in the mesoderm. Reese et $\mathrm{al}^{25}$ also suggested in the chicken embryos that antagonization of BMP signaling by noggin or chordin expressed in the notochord may be correlated with maintenance of the paired dorsal aortae. Thus, as candidate genes 


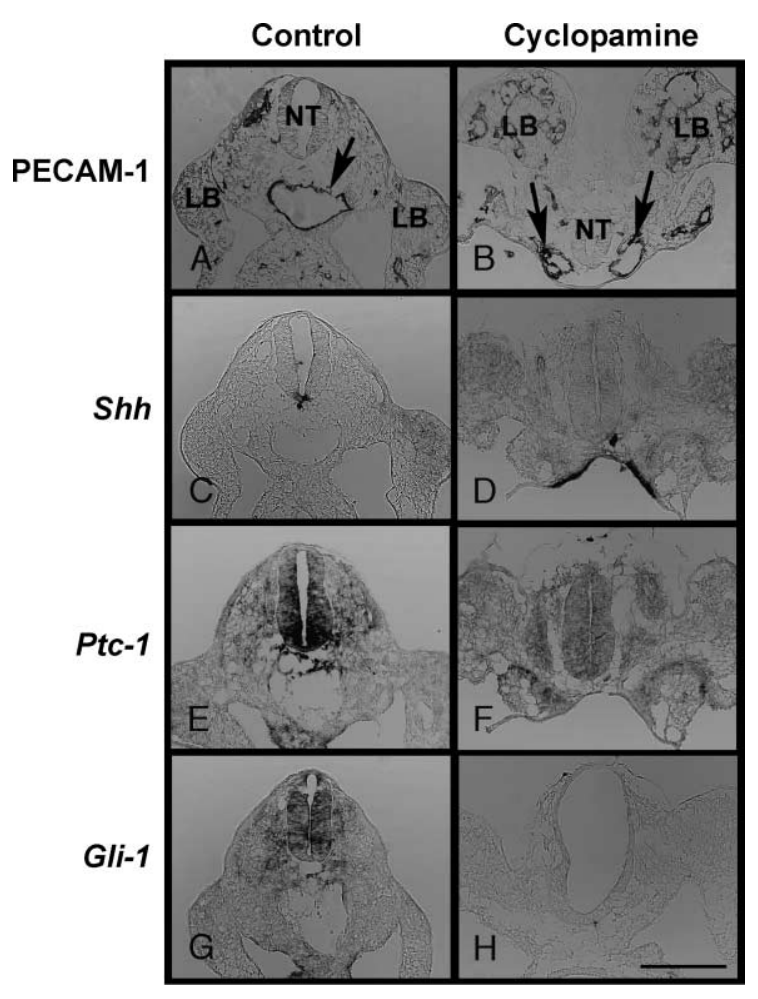

Fig 3 PECAM-1 immunostaining $(\mathrm{A}, \mathrm{B})$ and in situ hybridization of Shh (C, D), Ptc-1 (E, F) and Gli-1 (G, H) genes in the section at the limb bud level of the cultured $\mathrm{E} 9.5$ embryos of the control (A, C, E, G) and the cyclopaminetreated group $(B, D, F, H)$. (A) In the control group, the fused aorta is clearly detected ventral to the neural tube (NT) (arrow). (B) The aortae are separated in the cyclopaminetreated embryos (arrows). Note that the limb buds (LB) are dorsally situated in the cyclopamine group. (C) Shh is expressed in the notochord and the floor plate of the neural tube in the control group. (D) Shh is positive in the notochord (and the endoderm) in the cyclopmamine group. (E) Ptc-1 transcripts are found in the ventral neural tube and the surrounding mesenchyme in the control group. (F) Ptc-1 is also expressed in the same region of the cyclopamine-treated embryo, but its expression is significantly reduced. (G) Gli-1 mRNA is observed within and around the neural tube in the control group. (H) Gli-1 expression is nearly undetectable in the cyclopamine group. Bars, $200 \mu \mathrm{m}$.

downstream of Shh, we next performed VEGF and $B M P-4$ in situ hybridization in the embryos cultured for E8.0-9.5 with or without cyclopamine. VEGF transcripts were clearly detected in the neural tube of the control embryos (Fig 4A), as reported by Hogan et al. ${ }^{26}$ On the other hand, VEGF expression in the neural tube was almost undetectable in the cyclopamine-treated embryos (Fig 4B). BMP-4 expression was also detected in the lateral plate mesoderm ventral to the aorta in the control group (Fig 4C). In contrast, BMP-4 was not expressed in this portion in the cyclopamine-treated embryos (Fig 4D). Taken together, it was suggested that Shh signaling regulates $V E G F$ and $B M P-4$ expression in the midline surrounding the aorta, thereby mediating the aortic fusion.

\section{Craniofacial Vasculature was Perturbed by Blockade of Shh Signaling for E8.5-10.5}

We next examine the cultured embryos where Shh signaling was blocked from E8.5 (around somite stage 10 and thereafter). We collected the embryos about two days later at the stage of E10.5, because this period corresponds to our original protocol for holoprosencephaly model. ${ }^{8}$ In this condition, we previously reported defective angiogenic sprouting within the neural tube, ${ }^{20}$ and therefore we describe other vascular phenotypes, especially in the craniofacial region. As reported previously, the vessels on the surface of the craniofacial regions, possibly the external cardinal veins, were relatively dilated and filled with red blood cells just after culture. ${ }^{8}$ This

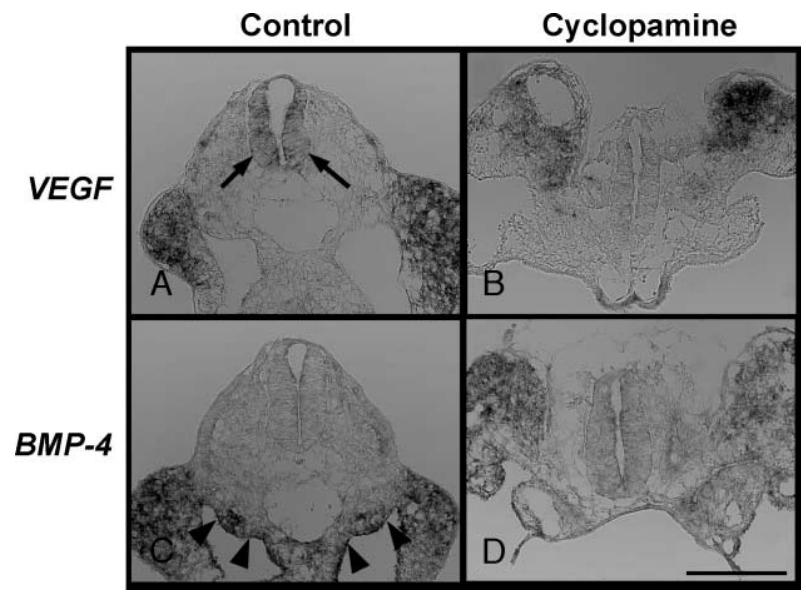

Fig 4 Expression of $\operatorname{VEGF}(\mathrm{A}, \mathrm{B})$ and $B M P-4(\mathrm{C}, \mathrm{D})$ genes in the E9.5 embryos of the control $(A, C)$ and the cyclopamine-treated group (B, D). (A) VEGF is expressed in the neural tube (arrows) and the limb buds in the control group. (B) VEGF expression in the neural tube is reduced in the cyclopamine-treated embryo. (C) BMP-4 transcripts are detected in the limb buds and the lateral plate mesoderm near the aorta (arrowheads) in the control group. (D) BMP4 expression is negative in the ventral region of the cyclopamine-treated embryos. Note that the VEGF and $B M P-4$ expression are maintained in the limb buds of the cyclopamine-treated embryos. Bar, $200 \mu \mathrm{m}$. 


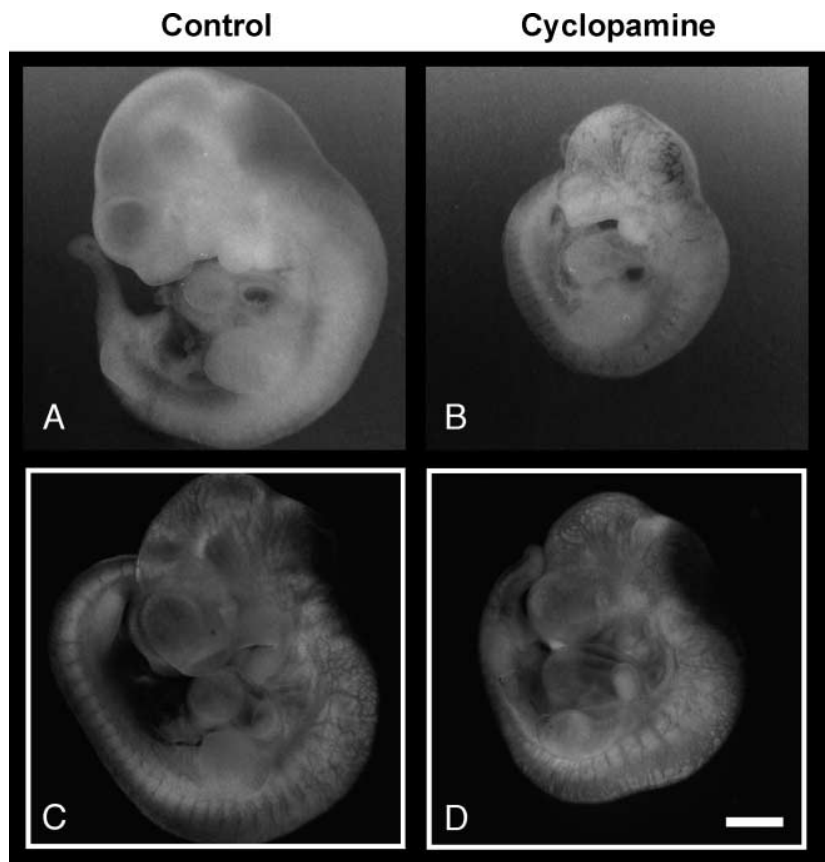

Fig 5 Plain appearances (A, B) and whole mount PECAM-1 immunostaining $(C, D)$ in the embryos cultured for E8.510.5 with $(\mathrm{B}, \mathrm{D})$ or without $(\mathrm{A}, \mathrm{C})$ cyclopamine. Note the extended craniofacial vasculature (possibly the external cardinal veins) filled with red blood cells in B. Bar, $500 \mu \mathrm{m}$.

phenotype was exaggerated in some selected cases (Figs 5A, B).

To observe vasculature of the embryo more clearly, we again conducted whole-mount immunohistochemistry against endothelial marker PECAM-1. Meticulous vascular networks were clearly observed in both the control and the cyclopamine-treated embryos (Figs 5C, D). The dilatation of the vessels was not so remarkable in the cyclopamine-treated group, possibly because fresh red blood cells filling the vessels were missing during immunostaining procedure. Close-up view of the immunostained embryos revealed that the vascular network in the craniofacial region of the cyclopamine embryos was coarser than that in the control embryos, especially in the forebrain (Figs 6A, B) and the midbrain (Figs 6D, E). Branches of the external cardinal veins were interrupted in some cases (Fig 6C). Cross sections of the craniofacial region showed that average diameter of the craniofacial vessels were greater in the cyclopamine-treated groups than in the control group (Figs 6F, G). These data suggested that the craniofacial vasculature had a tendency toward dilatation, interruption, and making a coarse network when treated with cyclopamine.

\section{DISCUSSION}

\section{Defects in Aortic Fusion}

Tn this study, we described two types of vascular 1 phenotype of the mouse embryos treated with blockers of hedgehog signaling. Administration of cyclopamine for E8.0 -E9.5 resulted in the defects in the process of aortic fusion. Later administration of cyclopamine for E 8.5-10.5 showed subtle vascular change: coarseness and slight dilatation of the vessels in the craniofacial region.

Vasculogenesis (de novo formation of vessels) of the embryonic aorta is observed as a form of the paired dorsal aortae through the entire length of the embryo, which later fuse with each other. ${ }^{10,11,27}$ The aortic fusion results in the single descending aorta and the bilateral aortic arches in the rostral trunk, which later remodel into disappearance of the right aortic arch and the single, left-sided aortic arch. ${ }^{11,27}$ Most previous descriptions of aortic morphogenesis focused on the aortic arches in relation to development of the pharyngeal arches, ${ }^{11,27,28}$ and the process of the aortic fusion have

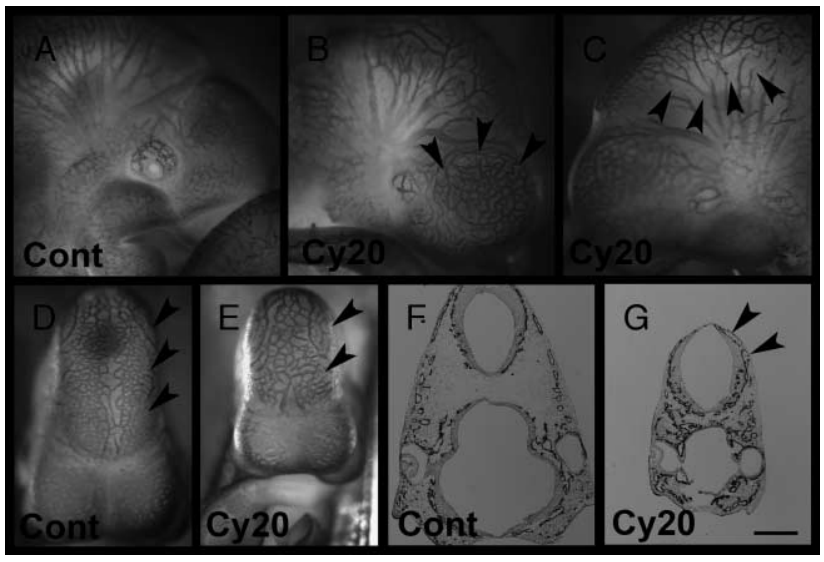

Fig 6 Close-up view of the craniofacial vasculature of the embryos cultured for E 8.5-10.5, with PECAM-1 immunostaining. $(\mathrm{A}-\mathrm{C})$ Oblique view of the craniofacial vasculature of the control $(\mathrm{A})$ and the cyclopamine-treated $(\mathrm{B}, \mathrm{C})$ embryos. The cyclopamine-treated embryos show coarser vascular network in the forebrain (arrowheads in B) than the control. Note interruptions of the external cardinal veins (arrowheads in C) seen in some embryos treated with cyclopamine. (D, E) Frontal view of the control (D) and the cyclopamine-treated (E) embryos. The midbrain vasculature is also coarser in the cyclopamine-treated embryos (arrowheads in E) than in the control (arrowheads in D). $(F, G)$ Craniofacial frozen sections of PECAM-1 immunohistochemistry in the control $(\mathrm{F})$ and the cyclopaminetreated $(\mathrm{G})$ embryos. Diameters of the vessels in the midbrain surface are larger in the cyclopamine-treated embryos (arrowheads in G) than in the control. Bar: $400 \mu \mathrm{m}$ (in F, G). 
been generally ignored and unappreciated. However, there are a few clinical reports of congenital aortic anomalies possibly derived from defects of the aortic fusion (i.e., duplication of the abdominal aortae). ${ }^{29}$ In this regard, our present findings may be of great importance unraveling molecular aspects of a previously unrecognized mechanism of the aortic fusion.

Vokes et al ${ }^{15}$ reported defects of the formation, but not of the fusion, of the paired dorsal aortae by cyclopamine treatment in the chicken embryos and Smo targeting in the mouse embryos. Although their results seem similar to our present data to some extent, their phenotype can be regarded as a defects in vasculogenesis in the slightly earlier developmental stages than ours. We also demonstrated that the VEGF and $B M P-4$ expressions in the midline were inhibited by cyclopamine, suggesting that the Shh-dependent aortic fusion is mediated by these genes. Lawson et $\mathrm{al}^{19}$ also reported that aortogenesis in the zebrafish embryo is mediated by VEGF downstream of Shh. Another group reported that ectopic application of exogenous VEGF near the dorsal aortae in the quail embryo promoted dense vascularization around the aortae, ${ }^{30,31}$ but they failed to exhibit the aortic fusion. On the other hand, Reese et $\mathrm{al}^{25}$ reported in the chicken embryos that BMP antagonists noggin and chordin were expressed in the notochord and that extirpation of the notochord resulted in fusion of the paired dorsal aortae. Considering their findings, we speculate that VEGF is an indirect mediator and BMP-4 regulates aortic fusion more directly in our experiment. Further studies are required addressing this point, such as in situ hybridization of noggin and chordin in the notochord, or overexpression or targeting of VEGF or BMPs.

\section{Defects in Craniofacial Vasculature}

We also observed angiogenic defects in the mouse embryos treated with cyclopmaine for E8.5-10.5. Although the phenotype in the craniofacial vasculature was very subtle - dilatation, interruption and coarseness of the network - there may be close association between these phenotypes and the holoprosencephalic morphology of these embryos. In this condition of cyclopamine administration, we previously found and reported defects of angiogenesis within the neural tube. ${ }^{20}$ Together with the aforementioned phenotype in the aortic fusion, our findings may be regarded as examples of Shh induced angiogenesis, which has been a recent topic in the field of vascular biology. ${ }^{15-19}$

There may be several ways to interpret mode of actions of Shh regarding this phenotype. A possibility of direct induction of angiogenesis by Shh should be firstly considered. However, we think this is not a case, because expression patterns of Shh-related genes in the craniofacial region ${ }^{8}$ have no relation to the location of the angiogenesis defects. The second possibility, thus, is indirect induction of craniofacial angiogenesis by Shh. In this case, Shh should induce angiogenic factors such as VEGF or angiopoietins in the craniofacial regions. We previously reported that angiogenesis within the neural tube is possibly mediated by the angiopoietin-1-positive motor neurons induced by Shh from the notochord or the floor plate. ${ }^{19}$ Further studies should be necessary for investigating whether such a mechanism is also found in the craniofacial regions. It should also taken into consideration that Shh signaling affects cranial neural crest cells, ${ }^{6,32}$ as shown in our previous embryonic models on holoprosencephaly and fetal alcohol syndrome. ${ }^{7,8}$ Recently, Etchevers et al. ${ }^{33}$ reported an unexpected contribution of neural crest cells to the smooth muscles and pericytes in the craniofacial vessel walls in the chicken embryos. These facts may imply the third interesting possibility of Shh-dependent recruitment of neural crest cells in the craniofacial angiogenesis for explaining our present results.

\section{Time-dependent Variations of the Holoprosencephaly-like Anomaly by Shh Signaling Inhibition: A Complex Cascade of Craniofacial Morphogenesis}

It is widely accepted that Shh has a pivotal role in craniofacial development and it is responsible for holoprosencephaly. ${ }^{1-3}$ Indeed, Shh knockout mice have a severe phenotype of cyclopia. ${ }^{4}$ However, one of the important problems yet to be fully elucidated is a mechanism of a wide spectrum of clinical manifestations of holoprosencephaly; from cyclopia to a normal face with only a single incisor. ${ }^{3}$ We considered time-dependent blockade of Shh signaling (a kind of conditional targeting) would be a possible approach addressing this problem, and cyclopamine administration in a narrow time-window in the whole embryo culture system is a simple method for achieving this purpose.

In this study, a wide variety of craniofacial phenotype could be obtained by different timing of cyclopamine administration, as reported by Cordero et $\mathrm{al}^{9}$ in chick embryos. Indeed, the phenotype was completely different between the group where cyclopamine was administered from E7.8 and the group from E8.5, and this fact may reflect dynamic complexity of craniofacial morphognesis during this period. The neuroepithelium, which is a simple plate at E8.0, becomes vesicular and eye primordia can be recognized 
at E8.5. Neural crest cells start to migrate in this stage to form the branchial arches at E8.7-9.0. Fundamental morphogenesis of the cardiovascular system, such as heart formation and the aortic fusion can be observed in the same developmental stage, and these morphogenetic processes may influence one another in a cascade-like manner.

We herein showed very different vascular phenotype due to cyclopamine administration for E8.09.5 and for E8.5-10.5, suggesting that mode of Shh induced angiogenesis is unique and distinct according to subtle difference of the developmental stage. Shh time-dependently influences morphogenesis of the central nervous system and viability of the neural crest cells as well. ${ }^{1,32}$ Thus, the holoprosencephaly-like morphological phenotype should be the final outcome and the sum of these Shh-dependent processes in the various tissues and their interrelationships. We consider this is one of the causative factors explaining a variety of clinical manifestations of holoprosencephaly; stochastic temporo-spatial fluctuations of Shh actions may be amplified and exaggerated through the complex craniofacial morphogenetic cascades to produce a wide range of clinical manifestations. In this sense, Shh-dependent angiogenesis reported in this study should be an integral part of the whole pathophysiological processes of holoprosencephaly.

The complexity of craniofacial morphogenesis is reflected in the other anomalies than holoprosencephaly. For example, it is known that various types of craniosynostoses with distinct symptoms such as Crouzon, Apert, Pfeiffer and Jackson-Weiss syndromes can be caused by mutations of the same gene fibroblast growth factor receptor II, and some of the mutations were identical among these different syndromes. ${ }^{34,35}$ A wide variety of phenotypes compared to genotype, as well as polygenic and multifactorial mode of etiologies, may be a common feature among craniofacial malformations. We consider that all of these are based on complex morphogenetic cascades of the craniofacial development.

\section{REFERENCES}

1. Cohen MM Jr. The hedgehog signaling network. Am J Med Genet 2003;123:A5-A28

2. Belloni E, Muenke M, Roessler E, et al. Identification of sonic hedgehog as a candidate gene responsible for holoprosencephaly. Nat Genet 1996;14:353-356

3. Roessler E, Belloni E, Gaudenz K, et al. Mutations in the human sonic hedgehog gene cause holoprosencephaly. Nat Genet 1996;14:357- 360

4. Chiang C, Litingtung $Y$, Lee E, et al. Cyclopia and defective axial patterning in mice lacking sonic hedgehog gene function. Nature 1996;383:407- 413
5. Hu D, Helms JA. The role of sonic hedgehog in normal and abnormal craniofacial morphogenesis. Development 1999; $126: 4873-4884$

6. Ahlgren SC, Thakur V, Bronner-Fraser M. Sonic hedgehog rescues cranial neural crest from cell death induced by ethanol exposure. Proc Natl Acad Sci U S A 2002;99:10476-10481

7. Yamada $Y$, Nagase T, Nagase M, et al. Gene expression changes of sonic hedgehog signaling cascade in a mouse embryonic model of fetal alcohol syndrome. J Craniofac Surg 2005;16: $1055-1061$

8. Nagase T, Nagase M, Osumi N, et al. Craniofacial anomalies of the cultured mouse embryo induced by inhibition of sonic hedgehog signaling: an animal model of holoprosencephaly. J Craniofac Surg 2005;16:80 - 88

9. Cordero D, Marcucio R, Hu D, et al. Temporal perturbations in sonic hedgehog signaling elicit the spectrum of holoprosencephaly phenotypes. J Clin Invest 2004;114:485 - 494

10. Coffin JD, Poole TJ. Embryonic vascular development: immunohistochemical identification of the origin and subsequent morphogenesis of the major vessel primordia in quail embryos. Development 1988;102:735-748

11. Moore KL, Persaud TVN. The developing human. Clinically oriented embryology, 7th. Philadelphia: W.B. Saunders, 2003

12. Drake CJ, Fleming PA. Vasculogenesis in the day 6.5 to 9.5 mouse embryo. Blood 2000;95:1671-1679

13. Kaufman MH. The atlas of the mouse development: Academic Press 1992

14. Wood HB, May G, Healy L, et al. CD34 expression patterns during early mouse development are related to modes of blood vessel formation and reveal additional sites of hematopoiesis. Blood 1997;90:2300-2311

15. Vokes SA, Yatskievych TA, Heimark RL, et al. Hedgehog signaling is essential for endothelial tube formation during vasculogenesis. Development 2004;131:4371-4380

16. Pola R, Ling LE, Silver M, et al. The morphogen sonic hedgehog is an indirect angiogenic agent upregulating two families of angiogenic growth factors. Nat Med 2001;7:706 -711

17. Pola R, Ling LE, Aprahamian TR, et al. Postnatal recapitulation of embryonic hedgehog pathway in response to skeletal muscle ischemia. Circulation 2003;108:479-485

18. Kusano KF, Allendoerfer KL, Munger W, et al. Sonic hedgehog induces arteriogenesis in diabetic vasa nervorum and restores function in diabetic neuropathy. Arterioscler Thromb Vasc Biol 2004;24:2102-2107

19. Lawson ND, Vogel AM, Weinstein BM. Sonic hedgehog and vascular endothelial growth factor act upstream of the notch pathway during arterial endothelial differentiation. Dev Cell 2002;3:127-136

20. Nagase T, Nagase M, Yoshimura K, et al. Angiogenesis within the developing mouse neural tube is dependent on sonic hedgehog signaling: possible roles of motor neurons. Genes Cells 2005;10:595 - 604

21. Nagase M, Nagase T, Koshima I, et al. Critical time window of hedgehog-dependent angiogenesis in murine yolk sac. Microvasc Res 2006

22. Nagase T, Sanai Y, Nakamura S, et al. Roles of HNK-1 carbohydrate epitope and its synthetic glucuronyltransferase genes on migration of rat neural crest cells. J Anat 2003;203:77- 88

23. Osumi N, Inoue T. Gene transfer into cultured mammalian embryos by electroporation. Methods 2001;24:35 - 42

24. Marigo V, Tabin CJ. Regulation of patched by sonic hedgehog in the developing neural tube. Proc Natl Acad Sci U S A 1996;93:9346 -9351

25. Reese DE, Hall CE, Mikawa T. Negative regulation of midline vascular development by the notochord. Dev Cell 2004; 6:699-708

26. Hogan KA, Ambler CA, Chapman DL, et al. The neural tube patterns vessels developmentally using the vegf signaling pathway. Development 2004;131:1503 - 1513 
27. Skandalakis JE, Gray SW, Symbas P. The thoracic and abdominal aorta. In: Skandalakis JE, Gray SW, eds. Embryology for surgeons. The embryological basis for the treatment of congenital anomalies, 2nd. Baltimore: Williams \& Wilkins, 1994:976-1030

28. Hiruma T, Nakajima Y, Nakamura H. Development of pharyngeal arch arteries in early mouse embryo. J Anat 2002;201:15-29

29. Takeshita T, Miyaji N, Kobayashi N, et al. A case of rare anomaly of the abdominal aorta: magnetic resonance image and angiographic finding. Nippon Igaku Hoshasen Gakkai Zasshi 1990;50:258 - 261

30. Poole TJ, Finkelstein EB, Cox CM. The role of FGF and VEGF in angioblast induction and migration during vascular development. Dev Dyn 2001;220:1-17
31. Finkelstein EB, Poole TJ. Vascular endothelial growth factor: a regulator of vascular morphogenesis in the Japanese quail embryo. Anat Rec 2003;272:A403 -A414

32. Ahlgren SC, Bronner-Fraser M. Inhibition of sonic hedgehog signaling in vivo results in craniofacial neural crest cell death. Curr Biol 1999;9:1304 - 1314

33. Etchevers HC, Vincent C, Le Douarin NM, et al. The cephalic neural crest provides pericytes and smooth muscle cells to all blood vessels of the face and forebrain. Development 2001;128:1059-1068

34. Gorlin RJ. Fibroblast growth factors, their receptors and receptor disorders. J Craniomaxillofac Surg 1997;25:69-79

35. Nagase T, Nagase M, Hirose $S$, et al. Mutations in Fibroblast growth factor receptor 2 gene and craniosynostotic syndromes in Japanese children. J Craniofac Surg 1998;9:162-170 